\title{
Experimental Evaluation of TCP BIC and Vegas in MANETs
}

\author{
M.Jehan \\ Associate Professor \\ Dept. of Computer Science \\ D.J.Academy For Managerial \\ Excellence, Coimbatore, India
}

\author{
G.Radhamani \\ Professor and Director \\ Dept. of Computer Science \\ Dr.G.R.Damodaran College of \\ Science, Coimbatore, India
}

\author{
T.Kalakumari \\ Assistant Professor \\ Dept. of Commerce and IT \\ Sri Krishna Arts and Science \\ College, Coimbatore, India
}

\begin{abstract}
The tremendous growth of wireless networks demands the need to meet different congestion control algorithms for wireless network. Usual transmission control protocol reduces its performance by misinterpreting mobility losses due to node motion as congestion losses in wireless mobility network. So the congestion control algorithms are important role in the communication networks. In this paper we have analyzed the TCP BIC and TCP Vegas congestion control algorithms and the performance of these algorithms through NS2 simulator with proper parameters.
\end{abstract}

\section{General Terms}

Wireless Networks, MANETs

\section{Keywords}

TCP Congestion Control Algorithms, MANET, BIC, Vegas.

\section{INTRODUCTION}

Mobile Ad hoc Networks (MANETs) are self-organizing wireless networks, in which also end nodes act as routers. MANET networking improves the efficiency and range of fixed and mobile internet access and enables totally new applications such as sensor networks. Its offer unique challenges and opportunities to network designers and administrators. They increase system capacity, reduce deployment cost as they require no supportive infrastructure, and reduce administrative cost as they are self configurable and self-adaptable. However, their inherent characteristic of frequent topology changes adds complexity to routing and transport connection management.

Although TCP provides reliable end-to-end delivery of data over wired networks, several recent studies have indicated that TCP performance degrades significantly in MANET [1][2][3]. In MANETs, the main problem of TCP lies in assuming every packet loss or delay exceeding retransmit timeout as a signal of indicating congestion in the network even though the source of loss or delay might be unrelated to congestion, such as delays due to reordering of packets in case of frequent route changes etc. Current MANET commercial applications have mainly been for military applications or emergency situations.

In this paper, we evaluated the Control Window (cwnd), Round Trip Delay Time (rtt), dropped packets ratio and throughput in TCP BIC and TCP Vegas.

This paper is organized as follows. In section 2, we describe the background and related work. Section 3 provides the network topology used and the simulations and the section 4 shows the simulation results. Finally we conclude this paper with future work.

\section{BACKGROUND AND RELATED WORK}

TCP uses a form of end-to-end flow control. In TCP, when a sender sends a packet, the receiver acknowledges receipt of the packet.

TCP BIC stand out from other TCP algorithms is its stability. It is a binary search algorithm where the window grows to the mid-point between the last window size (i.e., max) where TCP has a packet loss and the last window size (i.e., min) it does not have a loss for one RTT period.

The BIC TCP uses a Binary increase scheme to probe the available bandwidth efficiently [5], [6]. While reaching the high throughput, TCP BIC does not increase the RTT fairness problem of standard TCP [5].

TCP Vegas is a slow-start mechanism. TCP Vegas [8] controls its window size by observing RTTs of packets that the sender host has sent before. If observed RTTs become large, TCP Vegas recognizes that the network begins to be congested, and throttles the window size. Development work continues on TCP with congestion protocols such as BIC [7] and Vegas [8] being produced. There are many stream of investigation into TCP congestion carrying on such as Paganini et. al. [9] who model congestion based on provable mathematical modeling.

TCP Vegas was the first attempt to depart from the lossdriven paradigm of the TCP by introducing a mechanism of congestion detection before packet losses.

\section{THE SIMULATION}

\subsection{Network Topology and the Simulation in MANETs}

MANET topology will be expected to get change very dynamically, so we have only considered the performance of the congestion control algorithms for very short duration. That is, we assume that the proposed TCP communication will happen only for very short duration of time. And also we believe that the performance of the congestion algorithms during initial stage of the handshake will be very important in such short time TCP communication in a dynamically changing mobile ad hoc network topology. 


\section{Simulation Parameters}

The simulation area chosen is a random wireless mobile adhoc network topology, where 20 nodes are placed randomly. In this, the performance is measured up to $20 \mathrm{~m} / \mathrm{s}$ without node mobility, but after that all nodes are moving any direction in the wireless networks.

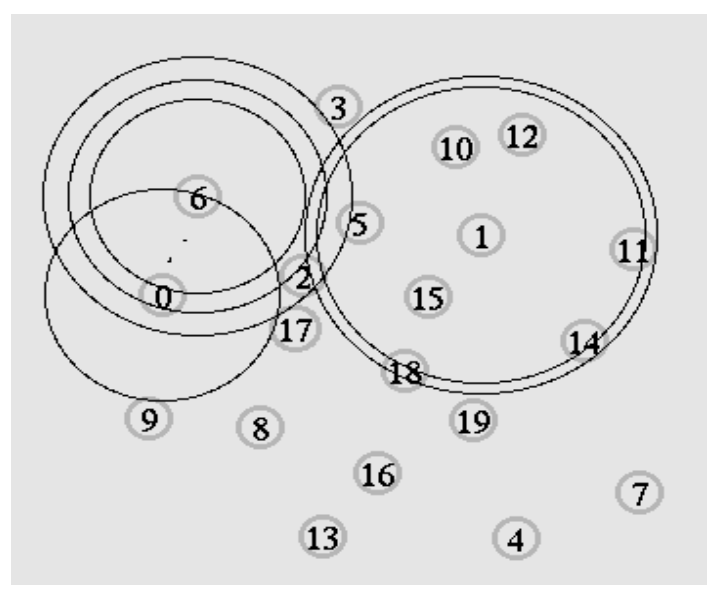

Fig 1: The Wireless Network Scenario

Number of Nodes

20

Number of Sending Nodes

Topography

$\mathrm{x}=500 \mathrm{y}=500$

Mobility

0 or $20 \mathrm{~m} / \mathrm{s}$

Mobility Start Time

20th Sec

Routing Protocol

AODV

Mac Type

802.11

Queue

Queue Size

DropTail/PriQueue

50

The Traffic Application FTP

TCP Packet Size

1448

TCP Initial Window Size 30000

\section{SIMULATION RESULTS}

In this section, we present the results of our ns-2 [19] simulations of both congestion control algorithms on wireless ad-hoc networks. This wireless simulation also has been run for 200 seconds. The graphs show the output of two ad-hoc network scenarios such as without mobility and the mobility after $20 \mathrm{~m} / \mathrm{seconds}$.

\subsection{Control Window in MANETs}

The following graphs show the control window size changes in MANETs

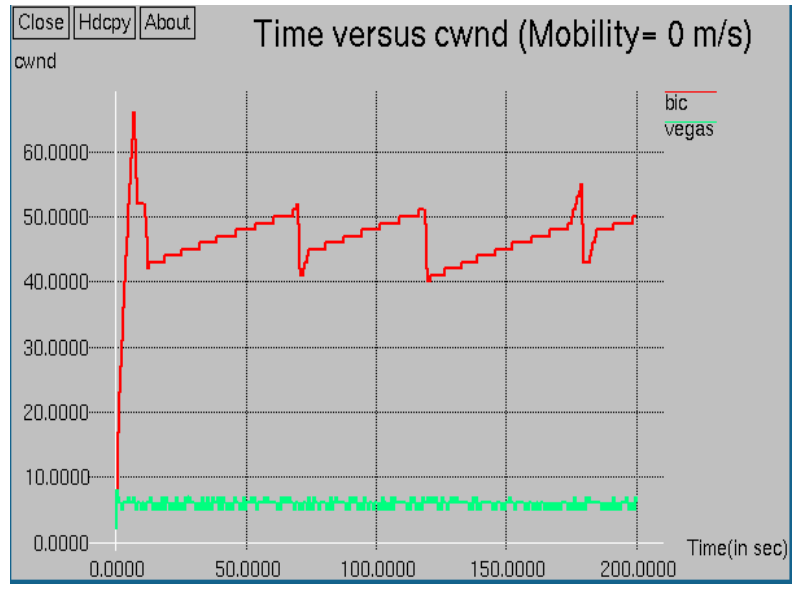

(2a)

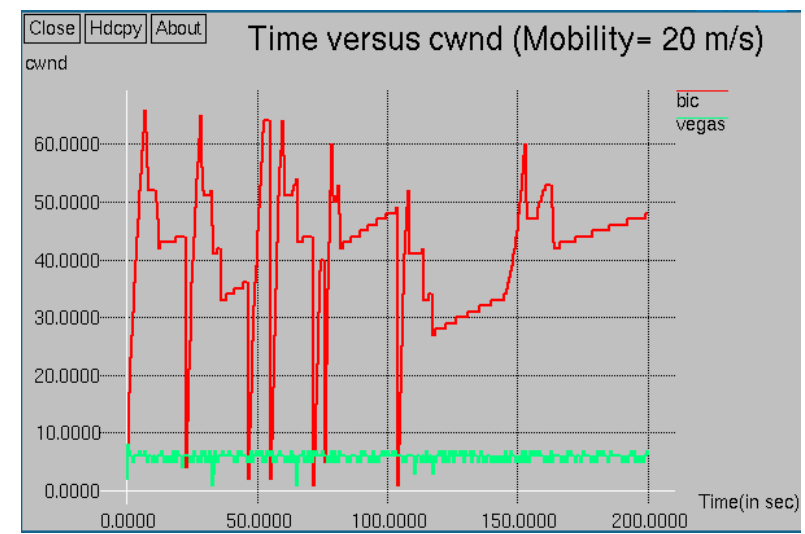

(2b)

Fig 2: The cwnd dynamics on Ad hoc Network (2a)Without Mobility (2b) With Mobility 20m/s

In the figure 2, the exponential window size increase, linear increase and drop-off occurs irregularly in BIC and TCP Vegas giving good result.

\subsection{Round Trip Delay Time in MANETs}

Figure $3 \mathrm{a}$ and $3 \mathrm{~b}$ prove that, the Round Trip Delay Time estimation of congestion control algorithms on wireless ad hoc networks with and without mobility. From the experimented result the TCP Vegas performance is better than TCP BIC.

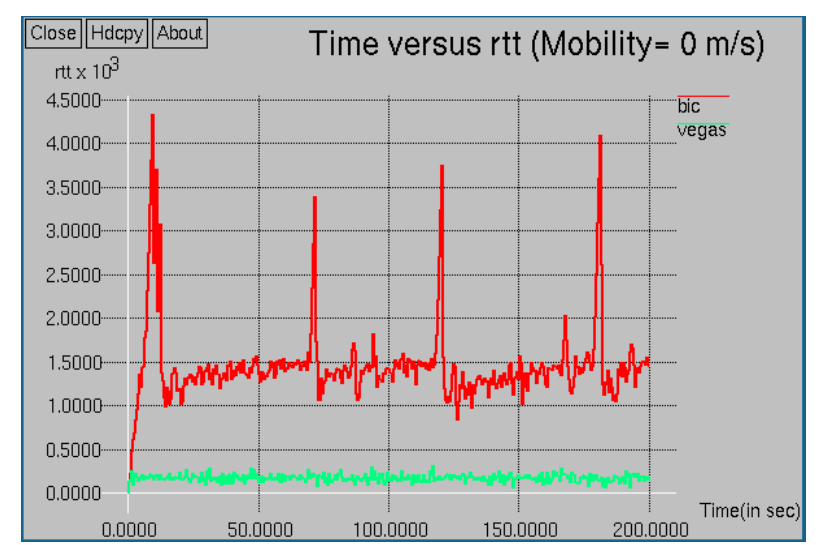

(3a) 


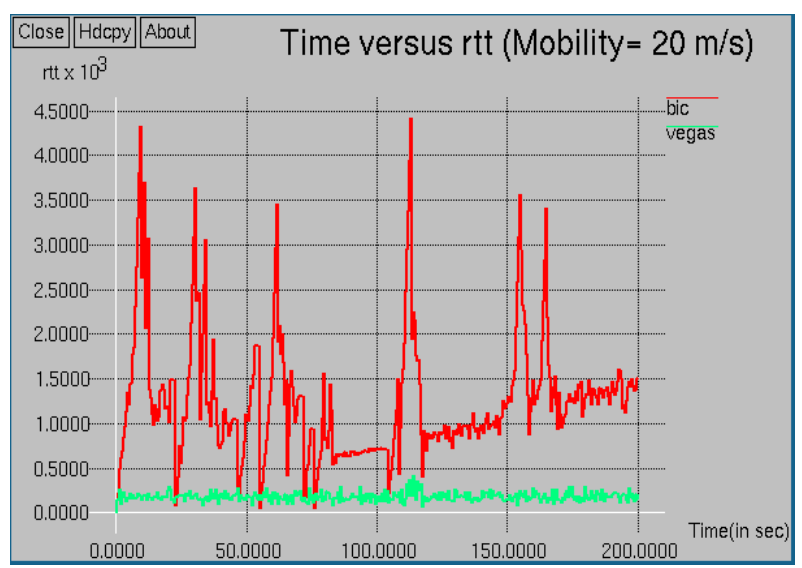

(3b)

Fig 3: The rtt dynamics on Ad hoc Network (3a) Without Mobility (3b) With Mobility

\subsection{Throughput over Time}

The throughput metric measures in the network can constantly provide data to the destination node. Throughput is the number of packet arriving at the sink per $\mathrm{ms} / \mathrm{second}$. Here we determine the instant throughput over time.

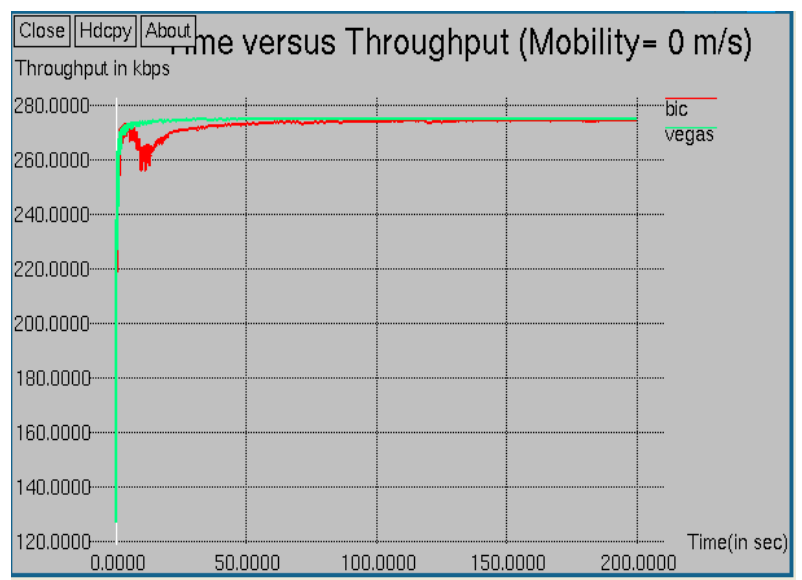

4(a)

Fig 4(a): Time versus Throughput without Mobility

As shown in the figure $4 \mathrm{a}$, both algorithms in the ad hoc networks without mobility, there was not much variation in throughput but at the same time the algorithm BIC shows little bit lower performance during the initial stage of the data transmission.

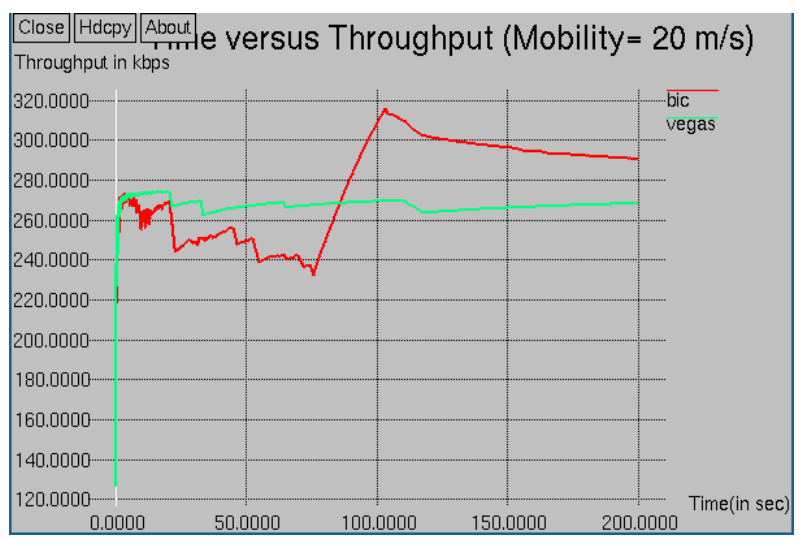

4(b)

Fig 4(b): Time versus Throughput with Mobility 20 m/s

In case of mobile scenario, as shown in the figure $4 \mathrm{~b}$, algorithm Vegas provided better throughput over time. Algorithm BIC provided best throughput after 75 seconds only but it provided deprived results during initial phase of the communication.

\subsection{Total Received Packets over Time}

To discriminate minor differences, here we account the total received packets as a cumulative summation of received packets at destination and record it over time.

As shown in the figure 5a, algorithms BIC and the Vegas in the non movement nodes stage, there was not much difference in total received packets.

But in the case of mobile circumstances, as shown in the figure $5 \mathrm{~b}$, the algorithm Vegas delivered more packets to the destination than BIC. The performance of Vegas is very much linear.

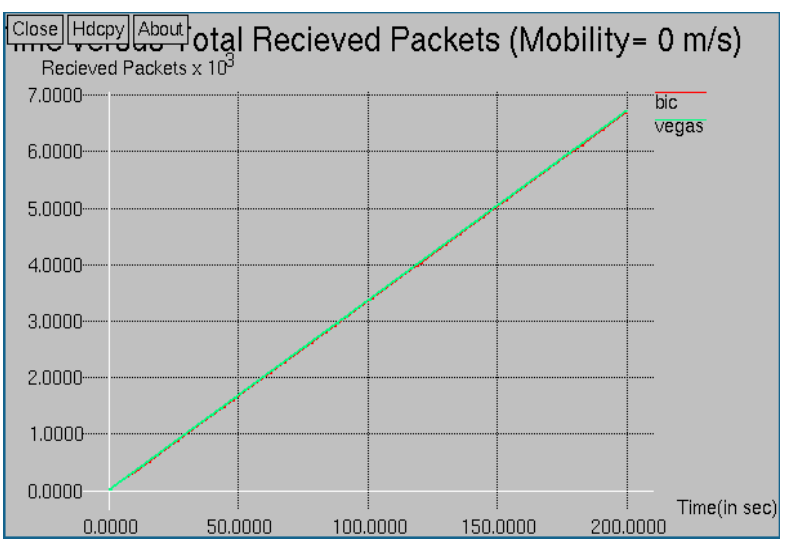

$5(a)$

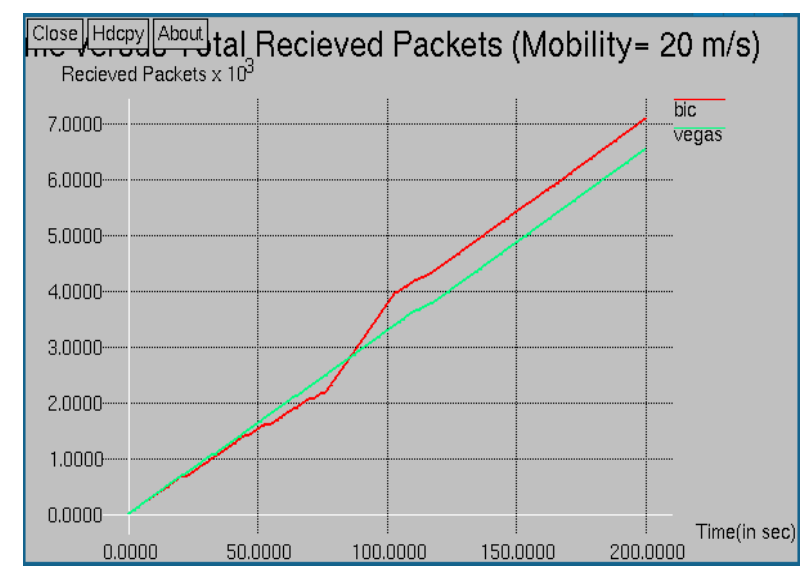

$5(\mathrm{~b})$

Fig 5: Total Received Packets (5a) Time versus Total Received packets without mobility (5b) Time versus total Received packets with mobility $20 \mathrm{~m} / \mathrm{s}$

\subsection{Dropped Packets over Time}

Several situations exist where source or destination devices drop packets due to heavy loads in the data transmissions of wireless communication. The dropped packet count is the number of data packets are collided or crashed during the data 
transmission between source and destination. Here we account the cumulative sum of dropped packets over time.

As shown in the figure 6(a), both algorithms BIC and Vegas are in the non movement nodes ad hoc networks, there was not much difference in the point of total dropped packets over time.

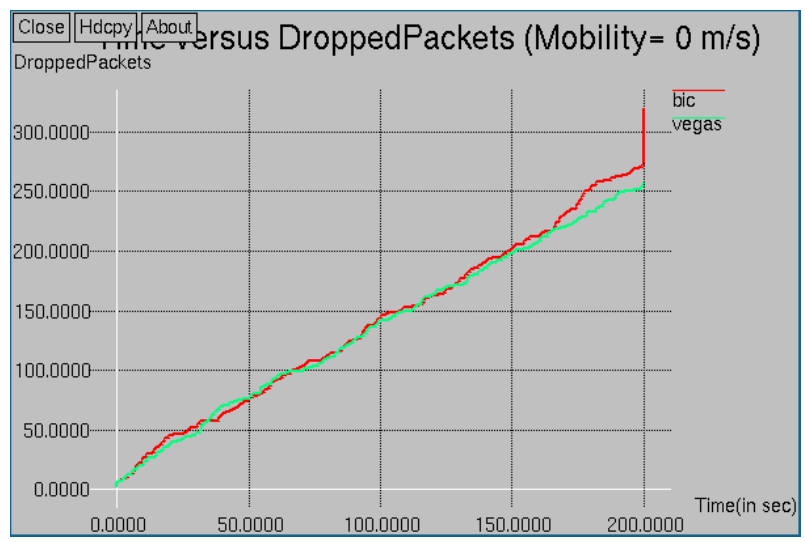

6(a)

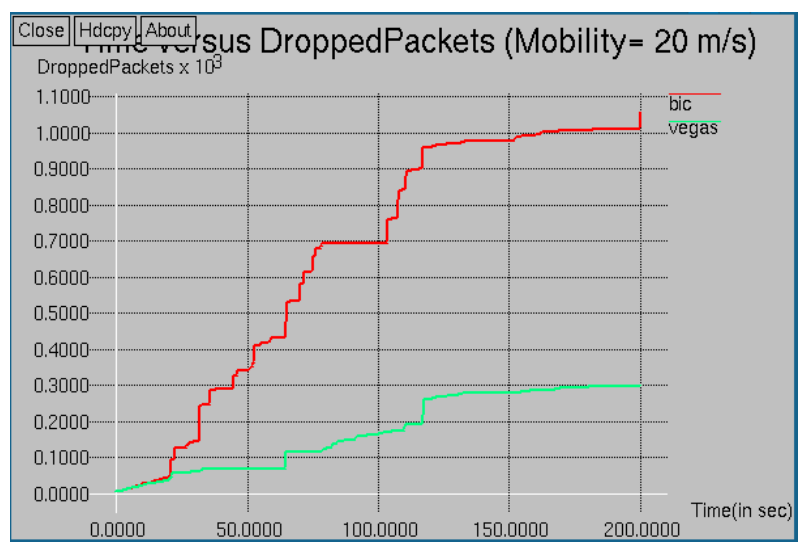

6(b)

Fig 6: Dropped packets (6a) Dropped packets without mobility (6b) Dropped packets with mobility $20 \mathrm{~m} / \mathrm{s}$

But in the case of mobile scenario, as shown in the figure 6(b), the algorithm Vegas provided the excellent result than BIC.

\subsection{Network Load over Time}

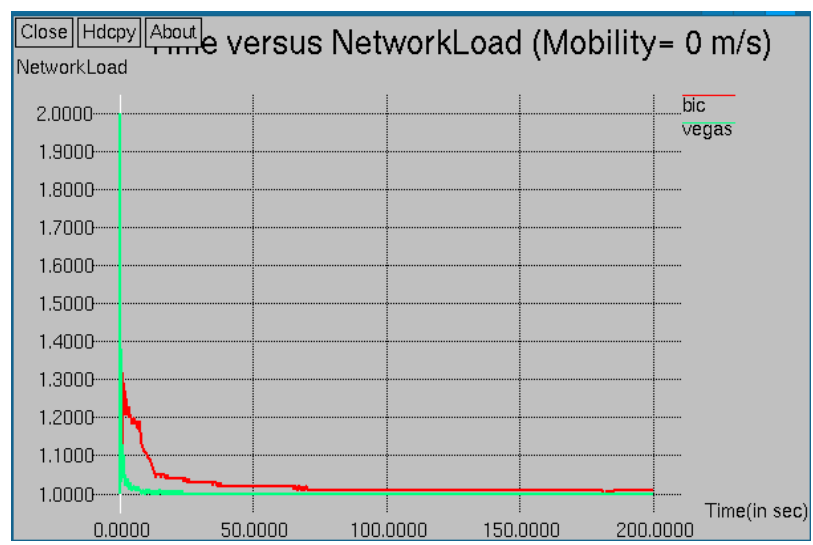

7(a)

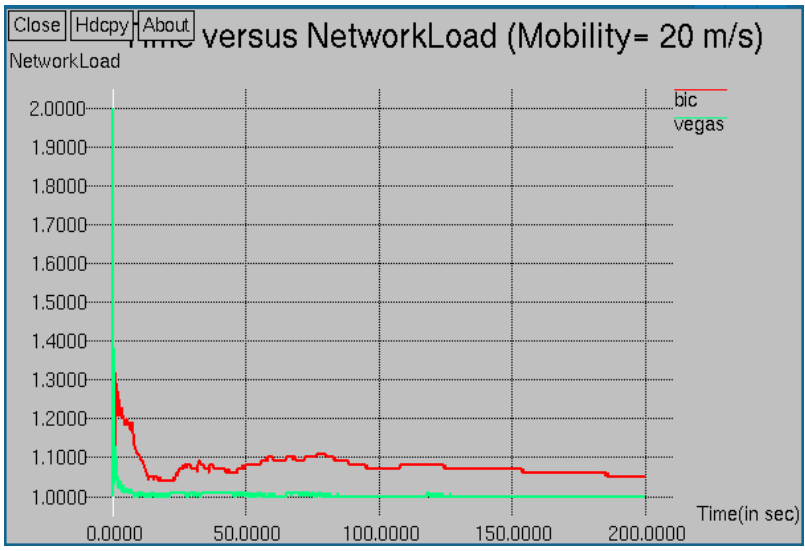

7(b)

Fig 7: Network Load (7a) Network Load without mobility (7b) Network Load with mobility $20 \mathrm{~m} / \mathrm{s}$

The term network load is used to describe how much data will be transmitted over a connection with in the time duration. It is a gross measurement, taking the total amount of data transferred in a given period of time as a rate, without taking into consideration the quality of the signal itself.

The ratio of the number of packets transmitted and the quantity of packets delivered to destination node within the time period.

As shown in the figure 7(a), algorithms in the scenario without mobility, there was not much divergence in total network load over time. But in the case of mobile scenario, as shown in figure 7(b), the algorithm Vegas provided very stumpy network load than the algorithm BIC.

\section{CONCLUSION}

We have successfully evaluated the TCP BIC and TCP Vegas congestion control algorithms using NS2 simulation tool. The performance of these two algorithms analyzed in ideal condition without any cross traffic and any other additional flows. In this small MANET scenario, the algorithm BIC provided good throughput after 75 seconds but algorithm Vegas provided stable and excellent result almost all over on the whole run time. So we conclude the algorithm Vegas will be the good algorithm for small and short duration communication.

\section{FUTURE WORK}

In this work, we have evaluated only two algorithms from the TCP congestion control algorithm group. We have planned to do another evaluation with based on the types of algorithms, such as slow start and Congestion Avoidance. In our future work will be few algorithms from each of this category for evaluation.

\section{ACKNOWLEDGMENT}

The authors thank the authorities of their working organization management for their support and the encouragement to pursue research in the chosen field of study.

\section{REFERENCES}

[1] G. Anastasi, M. Conti and E. Gregori, "IEEE 802.11 Ad Hoc Networks: Protocols, Performance and Open Issues", A book chapter, Ad Hoc Networking, Edited by S. Basagni, M. Conti, S. Giordano and I. Stojmenovic. 
IEEE Press and John Wiley and Sons, Inc, New York, 2004.

[2] I. Chlamtac, M. Conti, and J. Liu, "Mobile ad hoc networking: imperatives and challenges", Ad Hoc Networks Journal, vol.1, no. 1, pp. 13-64, Jul. 2003.

[3] A. Al Hanbali, E. Altman, P. Nain, "A Survey of TCP over Mobile Ad Hoc Networks", Research Report no. 5182, INRIA Sophia Antipolis research unit, May 2004.

[4] T. V. Lakshman, and U. Madhow, "The performance of TCP/IP for networks with high bandwidth-delay products and random loss", IEEE/ACM Transactions on Networking, vol. 5 no 3, pp. 336-350, July 1997.

[5] L. Xu, K. Harfoush, and I. Rhee, "Binary increase congestion control for fast, long distance networks". In Proceedings of IEEE INFOCOM, Hong Kong, Mar.2004.

[6] Yunhong Gu, Xinwei Hong, and Robert L. Grossman, "Experiences in Design and Implementation of a High Performance Transport Protocol", Supercomputing, Proceedings of the ACM/IEEE SC2004 Conference, pp. 22, 2004.

[7] Foez Ahmed, Sateesh Kumar Pradhan, Nayeema Islam, and Sumon Kumar Debnath, "Performance Evaluation of TCP over Mobile Ad-hoc Networks" in IJCSIS, Vol. 7, No. 1, 2010.

[8] Lawrence S. Brakmo and Larry L. Peterson, "TCP Vegas: End to end congestion avoidance on a global Internet," IEEE Journal on Selected Areas in Communications, vol.13, pp.1465-1480, October, 1995.

[9] Fernando Paganini, Zhikui Wang, John C. Doyle, and Steven H. Low. "Congestion control for high performance, stability, and fairness in general networks", IEEE/ACM Trans. Netw., 13(1):43-56, February 2005.

[10] S. Ryu, C. Rump, and C. Qiao, "Advances in Internet congestion control," IEEE Communications Surveys and Tutorials, vol. 3, pp. 28-39, 2003.
[11] Tomoya Hatano, Hiroshi Shigeno, Ken-ichi Okada, "TCP-friendly Congestion Control for High Speed Network", International Symposium on Applications and the Internet- SAINT'07, pp.10, 2007.

[12] K. Chandran, S. Raghunathan, S. Venkatesan, and R. Prakash," "A feedback based scheme for improving TCP performance in Ad-Hoc wireless networks", Proceedings of the International Conference on Distributed Computing Systems (ICDCS 98), Amsterdam, Netherlands, May 1998.

[13] T. S. Rappaport "Wireless Communications: Principles and Practice", Prentice-Hall, 1996.

[14] E. M. Royer and C. Perkins "An implementation study of the AODV routing protocol", In Proceedings of the IEEE Wireless Communications and Networking Conference, Chicago,IL, Sept. 2000.

[15] Kelly. T. "Scalable TCP: Improving performance in highspeed wide area networks", ACM SIGCOMM Computer Communication Review 33, 2 (April 2003), 83-91.

[16] Liu, S., Basar, T., and Srikant, R. "TCP-Illinois: A loss and delay-based congestion control algorithm for highspeed networks", In Proceedings of VALUETOOLS (Pisa, Italy, October 2006).

[17] R. K. Ahuja, T. L. Magnanti, and J. B. Orlin. "Network Flows, theory, Algorithms, and Applications", PrenticeHall, 1993.

[18] S. H. Bae, S.-J. Lee, and M. Gerla. "Unicast performance analysis of the ODMRP in a mobile ad-hoc network testbed", In Proceedings of IEEE ICCCN'2000, Las Vegas, USA, 2000.

[19] "ns-2 Network Simulator" http://www.isi.edu/nsnam/ns/, 2000. 